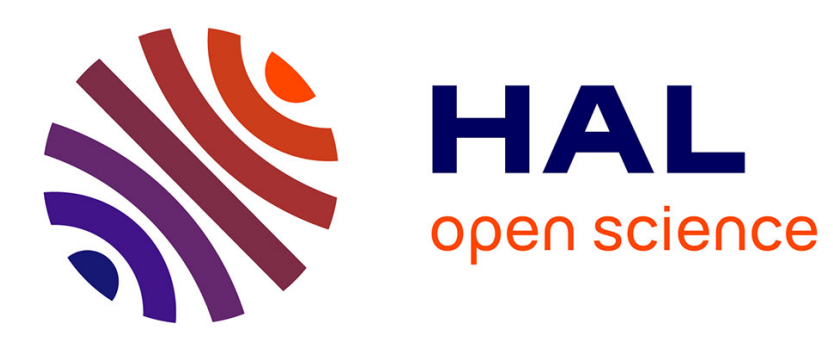

\title{
On the symmetric intersection of Rauzy fractals associated with the k-bonacci substitution
}

Hamdi Ammar, Julien Cassaigne, Tarek Sellami

\section{To cite this version:}

Hamdi Ammar, Julien Cassaigne, Tarek Sellami. On the symmetric intersection of Rauzy fractals associated with the k-bonacci substitution. Quaestiones Mathematicae, 2021, 44 (2), pp.199-216. 10.2989/16073606.2019.1680455 . hal-02375081

\section{HAL Id: hal-02375081 \\ https://hal.science/hal-02375081}

Submitted on 29 Dec 2020

HAL is a multi-disciplinary open access archive for the deposit and dissemination of scientific research documents, whether they are published or not. The documents may come from teaching and research institutions in France or abroad, or from public or private research centers.
L'archive ouverte pluridisciplinaire $\mathbf{H A L}$, est destinée au dépôt et à la diffusion de documents scientifiques de niveau recherche, publiés ou non, émanant des établissements d'enseignement et de recherche français ou étrangers, des laboratoires publics ou privés. 


\title{
ON THE SYMMETRIC INTERSECTION OF RAUZY FRACTALS ASSOCIATED WITH $k$-BONACCI SUBSTITUTION
}

\author{
HAMDI AMMAR, JULIEN CASSAIGNE, AND TAREK SELLAMI
}

\begin{abstract}
In this article, we study the intersection of Rauzy fractals associated with the $k$-bonacci substitution and its reversed substitution. Applying balanced pair algorithm to these two substitutions, we characterise all minimal balanced pairs, and we obtain a general formula for the associated intersection substitution. This substitution is defined over $\frac{k(k+1)}{2}$ letters.
\end{abstract}

\section{INTRODUCTION}

The symbolic dynamical system over three letters $\{1,2,3\}$ called Tribonacci substitution is defined as:

$$
\sigma: 1 \mapsto 12,2 \mapsto 13,3 \mapsto 1 .
$$

It was first studied by Gérard Rauzy in 1982 who associated to it a set known as Rauzy fractal. Rauzy's results have been generalized by Arnoux and Ito [3] for any unimodular Pisot irreducible substitutions $\sigma$ on $d$ letters.

Rauzy fractal is an important object in the study of dynamical systems associated to Pisot substitutions. In particular, it plays a fundamental role in the Pisot conjecture. Geometrical and topological properties of Rauzy fractals have been studied extensively; see, among other references, $[3,7,10$, $14,16,21,25,2]$.

Rauzy fractals appear naturally in connection with many topics such as numeration systems, geometrical representation of symbolic dynamical systems, multidimensional continued fractions and simultaneous approximations, self-similar tilings, and Markov partitions for hyperbolic automorphisms of the torus.

The $k$-bonacci substitutions are the Pisot substitutions of the form:

$$
\sigma_{k}: 1 \rightarrow 12,2 \rightarrow 13, \ldots,(k-1) \rightarrow 1 k, k \rightarrow 1 .
$$

For $k \geq 2$, the dynamical and geometrical properties of this family of substitutions have been studied in $[2,11,26,27]$.

Date: February 13, 2019.

2010 Mathematics Subject Classification. 28A80, 11B85, 37B10.

Key words and phrases. Substitutions, $k$-bonacci, balanced pairs algorithm, fractal sets. 
Our main focus in this paper is to study the intersection of Rauzy fractals associated to

$$
\sigma_{k}:\left\{\begin{array}{l}
1 \mapsto 12 \\
2 \mapsto 13 \\
\vdots \\
(k-1) \mapsto 1 k \\
k \mapsto 1
\end{array} \quad \text { and } \quad \sigma_{k}^{*}:\left\{\begin{array}{l}
1 \mapsto 21 \\
2 \mapsto 31 \\
\vdots \\
(k-1) \mapsto k 1 \\
k \mapsto 1
\end{array}\right.\right.
$$

over the alphabet $\mathcal{A}=\{1,2, \ldots, k\}$, where $k \geq 2$. The two substitutions $\sigma_{k}$ and $\sigma_{k}^{*}$ have the same incidence matrix.

The fixed point of the substitution $\sigma_{k}$ is a generalization of the Fibonacci word to the $k$-letter alphabet. It is the unique fixed point $u=u_{0} u_{1} \ldots$, of this Pisot substitution. For more details see [16].

The study of intersection of the Rauzy fractals was initiated by Sing and Sirvent [22]. They studied the prefix-suffix automata associated with each substitution and considered the product automaton to obtain common points belonging to the intersection of the Rauzy fractals. They studied a sequence of dynamical systems defined on sets $\mathrm{F}_{k}$, included in the common dynamics of irreducible Pisot substitutions with the same incidence matrix. These subsets have zero Lebesgue measure. For more details see [22].

Sellami [17, 18], under the Pisot condition, characterized the intersection of interiors of two Rauzy fractals associated with two unimodular irreducible Pisot substitutions $\tau_{1}$ and $\tau_{2}$ with the same incidence matrix. He proved that if the origin is an exclusive inner point of the Rauzy fractal associated with the substitution $\tau_{1}$, then the intersection has nonempty interior and has positive Lebesgue measure. He showed, under the Pisot conjecture, that the closure of the intersection of the interior of two Rauzy fractals is a substitutive set. This means that the intersection can be seen as a new Rauzy fractal associated with a new substitution, obtained by the balanced pair algorithm.

In this paper, we apply the balanced pairs algorithm to the substitutions $\sigma_{k}$ and $\sigma_{k}^{*}$. We characterize explicitly the substitution for intersection, defined on $\frac{k(k+1)}{2}$ letters for $k \geq 2$.

This paper is organized as follows. In Section 2, we give some basic notions and we explain the construction of Rauzy fractal associated with an irreducible Pisot substitution. In Section 3, first, we recall the definition of the balanced pair algorithm. Then, we recall the way to obtain the substitution associated with the intersection of the Rauzy fractals by application of the balanced pair algorithm. Section 4, will be about the application of the balanced pair algorithm to the $k$-bonacci substitution and its reversed substitution to obtain a general formula for the associated intersection substitution. Finally, in section 5, we present some examples. 


\section{Substitutions AND RAUZY FraCtals}

2.1. Substitutions. Let $\mathcal{A}$ be a finite set of letters, called alphabet. In particular, let us denote $\mathcal{A}=\{1, \ldots, k\}$ where $k$ is a positive integer. We denote by $\mathcal{A}^{*}=\bigcup_{i>0} \mathcal{A}^{i}$ the free monoid generated by $\mathcal{A}$ by concatenation, that is, the set of finite words over the alphabet $\mathcal{A}$ with the operation of concatenation. We denote by $\varepsilon$ the empty word.

Definition 1. (Substitution) A substitution $\sigma$ over the alphabet $\mathcal{A}$ is a map $\sigma: \mathcal{A} \rightarrow \mathcal{A}^{*}$, such that for any letter $j, \sigma(j)$ is a non-empty word. The substitution $\sigma$ over $\mathcal{A}$ is extended to an endomorphism of $\mathcal{A}^{*}$ by concatenation, i.e. $\sigma(\varepsilon)=\varepsilon$ and $\sigma(U V)=\sigma(U) \sigma(V)$, for all words $U, V \in \mathcal{A}^{*}$.

For any word $U \in \mathcal{A}^{*}$, let $|U|$ be the length of the word $U$. For any letter $j \in \mathcal{A},|U|_{j}$ denotes the number of occurrences of $j$ in the word $U$. We denote by $[\sigma(i)]_{j}$ the $j$-th symbol of the word $\sigma(i)$, i.e.,

$$
\sigma(i)=[\sigma(i)]_{1} \ldots[\sigma(i)]_{|\sigma(i)|} .
$$

For a finite word $u=u_{1} u_{2} \cdots u_{r}$, the reversal of $u$ is the word

$$
u^{*}=u_{r} u_{r-1} \cdots u_{1} .
$$

Definition 2. A finite word $u$ is said to be a Palindrome if

$$
u^{*}=u \text {. }
$$

The Abelianization map is the map $f: \mathcal{A}^{*} \rightarrow \mathbb{N}^{k}$ defined by $f(w)=$ $\left(|w|_{1},|w|_{2}, \ldots .,|w|_{k}\right)$.

Definition 3. (Incidence matrix) Let $\sigma$ be a substitution over $\mathcal{A}=\{1, \ldots, k\}$. Its incidence matrix $M_{\sigma}=\left(m_{i, j}\right)_{1 \leq i, j \leq k}$ is defined as the square matrix with entries $m_{i, j}=|\sigma(j)|_{i}$, for all $i, j$.

Note that $f(\sigma(w))=M_{\sigma} f(w)$ for all $w \in \mathcal{A}^{*}$.

Definition 4. A substitution is said to be unimodular if $\operatorname{det}\left(M_{\sigma}\right) \in\{-1,+1\}$.

Definition 5. (Primitive substitution) A substitution is said to be primitive if its incidence matrix is primitive, i.e., there exists a power of its incidence matrix whose entries are all positive.

In other words, definition 5 is equivalent to having a positive naturel number $t$ such that for all $i, j \in \mathcal{A}$ the letter $j$ appears in the word $\sigma^{t}(i)$.

Remark 2.1. If $\sigma$ is primitive, the Perron-Frobenius Theorem asserts that $M_{\sigma}$ has a simple positive eigenvalue $\lambda$, that is larger than the absolute value of all other eigenvalues.

Let $\mathcal{A}^{\mathbb{N}}$ (respectively $\mathcal{A}^{\mathbb{Z}}$ ) denote the set of one-sided (respectively twosided) infinite sequences on $\mathcal{A}^{\mathbb{Z}}$. The map $\sigma$ is extended in a natural way to 
$\mathcal{A}^{\mathbb{N}}$ and $\mathcal{A}^{\mathbb{Z}}$. Let $u=\ldots u_{-1} \cdot u_{0} u_{1} \ldots$ be an element of $\mathcal{A}^{\mathbb{Z}}$, where the dot is used to denote the zeroth position. So, $\sigma(u)$ is of the form:

$$
\ldots\left[\sigma\left(u_{-1}\right)\right]_{1} \ldots\left[\sigma\left(u_{-1}\right)\right]_{\left|\sigma\left(u_{-1}\right)\right|} \cdot\left[\sigma\left(u_{0}\right)\right]_{1} \ldots\left[\sigma\left(u_{0}\right)\right]_{\left|\sigma\left(u_{0}\right)\right|}\left[\sigma\left(u_{1}\right)\right]_{1} \ldots
$$

A fixed point of $\sigma$ is an infinite word $u$ in $\mathcal{A}^{\mathbb{N}}$ (or in $\mathcal{A}^{\mathbb{Z}}$ ) such that $\sigma(u)=u$. A periodic point of $\sigma$ is an infinite word $u$ in $\mathcal{A}^{\mathbb{N}}$ (or in $\mathcal{A}^{\mathbb{Z}}$ ) such that $\sigma^{s}(u)=u$ for some $s>0$. It can be proved that every substitution admits a periodic point [15]. If $\sigma$ has no fixed point, we consider a power of $\sigma$, that have a fixed point.

Remark 2.2. When $\sigma$ is primitive, then exists a finite number of periodic points (see [9]).

Definition 6. Let $\sigma^{*}: \mathcal{A} \rightarrow \mathcal{A}^{*}$ be the reverse substitution of $\sigma$, defined as follows:

$$
\left[\sigma^{*}(i)\right]_{j}=[\sigma(i)]_{|\sigma(i)|-j+1} \text { and }\left|\sigma^{*}(i)\right|=|\sigma(i)| .
$$

Let $\sigma$ be a substitution such that it has a fixed point $u=\ldots u_{-1} \cdot u_{0} u_{1} \ldots$ Let $\sigma^{*}$ be the reverse substitution of $\sigma$. Then, $\sigma^{*}$ has a fixed point $u^{*}=$ $\ldots u_{-1}^{*} \cdot u_{0}^{*} u_{1}^{*} \ldots$, with the property $u^{*}:=u_{-i-1}$, for $i \in \mathbb{Z}$ (see for more details $[20])$.

Let $u$ be a fixed point of $\sigma$, we associate to $u$ the dynamical system $\left(\Omega_{u}, S\right)$, where $S$ is the shift map on $\mathcal{A}^{\mathbb{N}}$ given by $S\left(w_{0} w_{1} w_{2} \ldots\right)=w_{1} w_{2} \ldots$ (respectively $S(w)=v$, where $\left.v_{i}=w_{i+1}\right)$ and $\Omega_{u}$ is the closure of $\left\{S^{m}(u): m \geq 0\right\}$ in $\mathcal{A}^{\mathbb{N}}$ equipped with the product topology.

Let us recall that an algebraic integer $\beta>1$ is a Pisot number if all its algebraic conjugates $\beta^{(j)}$ other than $\beta$ itself satisfy $\left|\beta^{(j)}\right|<1$.

Definition 7. (irreducible Pisot substitution) A substitution is Pisot irreducible if the dominant eigenvalue of its incidence matrix is a Pisot number, and if the characteristic polynomial of the incidence matrix is irreducible. Any irreducible Pisot substitution is primitive [7].

There is a long-standing conjecture stating that the dynamical system associated to an unimodular irreducible Pisot substitution is measurably conjugate to a translation on a $(k-1)$-dimensional torus ( $\mathrm{cf}[16,30])$. This conjecture is known in the literature as the Pisot conjecture. Rauzy approached it via geometrical realization of the symbolic system. He proved it in the case of the Tribonacci substitution, $\sigma(1)=12, \sigma(2)=13$ and $\sigma(3)=1$ (cf. [16]). In his proof, the construction of a planar set in $\mathbb{R}^{3}$, and of a $k$-1-dimensional set in $\mathbb{R}^{k}$ (for irreducible substitutions). This set is known as the Rauzy fractal associated with the substitution. For references on conditions under which the Pisot conjecture is true, we refer to, among other references $[1,3,4,5,6,7,12,13,14,16,29,2,30]$. 
2.2. Rauzy fractals. In order to define Rauzy fractals, we present the following constructions and notations.

Let $\sigma$ be an unimodular Pisot substitution and $\lambda$ the Perron-Fobenius eigenvalue of the incidence matrix $M_{\sigma}: \lambda$ is a Pisot number. The characteristic polynomial of $M_{\sigma}$ might be reducible, so the algebraic degree of $\lambda$ is smaller than or equal to $k$, the cardinality of the alphabet $\mathcal{A}$. We decompose $\mathbb{R}^{k}$ into a direct sum of subspaces, determined by the eigenvalues of $M_{\sigma}$. In particular, we consider: $\mathbf{H}_{u}$ the $\lambda$-expanding space of $M_{\sigma}$, the eigenspace associated with the eigenvalue $\lambda, \mathbf{H}_{s}$ as the $\lambda$-contracting space of $M_{\sigma}$, the sum of the eigenspaces associated with the Galois conjugates of $\lambda$ and $\mathbf{H}_{e}$ as the complementary space, i.e., the direct sum of the eigenspaces associated with the remaining eigenvalues of $M_{\sigma}$.

Hence, by the definition of the subspaces, we have $\mathbb{R}^{k}=\mathbf{H}_{u} \oplus \mathbf{H}_{s} \oplus \mathbf{H}_{e}$. The space $\mathbf{H}_{e}$ is trivial if and only if the substitution is irreducible.

Let $\Pi_{s}: \mathbb{R}^{k} \rightarrow \mathbf{H}_{s}$ be the linear projection into $\mathbf{H}_{s}$ along $\mathbf{H}_{u} \oplus \mathbf{H}_{e}$.

Definition 8. A stepped line $L=\left(x_{n}\right)_{n \geq 0}$ in $\mathbb{R}^{k}$ is a sequence (finite or infinite) of points such that the steps $x_{n+1}-x_{n}$ belong to a finite set. A canonical stepped line is a stepped line such that $x_{0}=0$ and for all $n \geq 0$, $x_{n+1}-x_{n}$ belongs to the canonical basis of $\mathbb{R}^{k}$.

Using the Abelianization map to any finite or infinite word $U$, we can associate a canonical stepped line in $\mathbb{R}^{k}$ as the sequence $\left(f\left(P_{i}\right)\right)$, where $P_{i}$ is the prefix of length $i$ of $U$.

2.3. Definition of Rauzy fractal. The canonical stepped line associated with a periodic point of an irreducible Pisot substitution is that it remains within bounded distance from the expanding direction given by the PerronFrobenius eigenvector of the incidence matrix $M_{\sigma}$ (see [3]). In the reducible case, the discrete line may have other expanding directions. However, the projection of the discrete line by $\Pi_{s}$ still provides a bounded set. For more details, we refer to [8].

Definition 9. Let $\sigma$ be a primitive unimodular Pisot substitution with dominant eigenvalue $\lambda$. The Rauzy fractal (or central tile) associated with $\sigma$ is the closure of the projection of the vertices of the canonical stepped line associated with any periodic point $u=\left(u_{d}\right)_{d \in \mathbb{N}}$ of $\sigma$ on the $\lambda$-contracting space $\mathbf{H}_{s}$. We will denote it by $\mathcal{R}_{\sigma}$, such that

$$
\mathcal{R}_{\sigma}=\overline{\left\{\Pi_{s}\left(f\left(u_{0} \ldots u_{d-1}\right)\right), d \in \mathbb{N}\right\}} .
$$

Subtiles of the central tile $\mathcal{R}_{\sigma}$ are naturally defined, depending on the letter associated with the vertex of the stepped line that is projected. One thus gets for any $i \in A$,

$$
\mathcal{R}_{\sigma}(i)=\overline{\left\{\Pi_{s}\left(f\left(u_{0} \ldots u_{d-1}\right)\right), d \in \mathbb{N}, u_{d}=i\right\}} .
$$



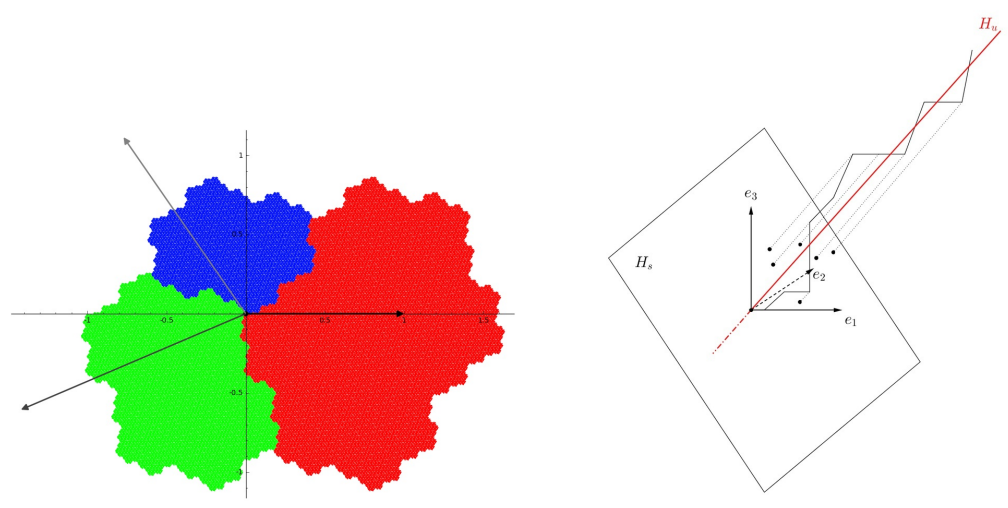

FiguRE 1. The projection method to get the Rauzy fractal.

Remark 1. It follows from the primitivity of the substitution $\sigma$ that the definition of $\mathcal{R}_{\sigma}$ and $\mathcal{R}_{\sigma}(i)(i \in \mathcal{A})$ does not depend on the choice of the periodic point $u \in \mathcal{A}^{\mathbb{N}}$ ([3]).

By definition, the central tile $\mathcal{R}_{\sigma}$ consists of the union of its subtiles, i.e.,

$$
\mathcal{R}_{\sigma}=\bigcup_{i \in \mathcal{A}} \mathcal{R}_{\sigma}(i)
$$

\section{BALANCED PAIR ALGORITHM AND INTERSECTION OF RAUZY}

FRACTALS

The description of the common dynamics for two substitutions $\tau_{1}$ and $\tau_{2}$ that have the same incidence matrix is a significant issue. It was previously tackled in $[18,22,24]$. The technique, which is based on the balanced pair algorithm for two substitutions having the same incidence matrix, is used by Sellami in $[17,18]$. This algorithm is a variation of the classical balanced pair algorithm introduced by Livshits [13] in the context of the Pisot conjecture, fore more details see for instance [28]. The version of the balanced pair algorithm introduced by Sellami will be used in this article and described in the following section.

3.1. Balanced pair algorithm. We explain the balanced pair algorithm for two substitutions $\tau_{1}$ and $\tau_{2}$ having the same incidence matrix. We shall assume that the substitutions are primitive. This algorithm was introduced in [17] and [18] in the context of the study of common dynamics associated with $\tau_{1}$ and $\tau_{2}$.

Definition 10. Let $(U, V) \in \mathcal{A}^{*} \times \mathcal{A}^{*}$ a pair of finite words. We say that $\left(\begin{array}{l}U \\ V\end{array}\right)$ is a balanced pair, if $f(U)=f(V)$. 
Definition 11. Let $U \in \mathcal{A}^{*}$ and $\langle U\rangle_{m}$ be the prefix of $U$ of length $m$. A minimal balanced pair is a balanced pair $\left(\begin{array}{c}U \\ V\end{array}\right)$ if $f\left(\langle U\rangle_{m}\right) \neq f\left(\langle V\rangle_{m}\right)$, for all $1 \leq m<|U|$.

Definition 12. The concatenation of two balanced pairs $\left(\begin{array}{c}X \\ Y\end{array}\right)$ and $\left(\begin{array}{c}U \\ V\end{array}\right)$ is the balanced pair defined by:

$$
\left(\begin{array}{c}
X \\
Y
\end{array}\right)\left(\begin{array}{c}
U \\
V
\end{array}\right)=\left(\begin{array}{c}
X U \\
Y V
\end{array}\right)
$$

Let $\tau_{1}$ and $\tau_{2}$ be two irreducible Pisot substitutions with the same incidence matrix. Let $u$ and $v$ be elements of $\mathcal{A}^{\mathbb{N}}$ that are periodic points of $\tau_{1}$ and $\tau_{2}$ respectively. The balanced pair algorithm gives a decomposition of the double periodic points $\left(\begin{array}{l}u \\ v\end{array}\right)$ into minimal balanced pairs.

What we are looking for is when we have the number of minimal balanced pairs is bounded.

The balanced pair algorithm for $\left(\begin{array}{l}u \\ v\end{array}\right)$ is defined as follows:

At first, let $\left(\begin{array}{c}u_{0} \\ v_{0}\end{array}\right)$ be the first minimal balanced pair, where $u_{0}, v_{0}$ are prefixes of $u, v$ respectively. We apply the substitutions $\tau_{1}$ and $\tau_{2}$ to this balanced pair in the following way $\left(\begin{array}{l}u_{0} \\ v_{0}\end{array}\right) \rightarrow\left(\begin{array}{c}\tau_{1}\left(u_{0}\right) \\ \tau_{2}\left(v_{0}\right)\end{array}\right)$. Since the substitutions $\tau_{1}$ and $\tau_{2}$ have the same incidence matrix, the pair $\left(\begin{array}{c}\tau_{1}\left(u_{0}\right) \\ \tau_{2}\left(v_{0}\right)\end{array}\right)$ is balanced. Then, we decompose this new balanced pair into minimal balanced pairs. We repeat this procedure for each of these new minimal balanced pairs.

With this decomposition, we obtain all the common points of the two stepped lines associated with the two periodic points $u$ and $v$. This means that we obtain common points from the interior of the two Rauzy fractals of $\tau_{1}$ and $\tau_{2}$. If the substitutions $\tau_{1}$ and $\tau_{2}$ verify the Pisot conjecture and 0 is an exclusive inner point of their Rauzy fractal, the set of minimal balanced pairs is finite and the algorithm terminates (cf.[18]). In this case, we set up $E$ as the set of minimal balanced pairs obtained in the algorithm. We define a new substitution $\Sigma$ over the alphabet of minimal balanced pairs, $\Sigma: E^{*} \rightarrow E^{*}$ such that, for all $\left(\begin{array}{c}U \\ V\end{array}\right) \in E$

$$
\Sigma\left(\left(\begin{array}{c}
U \\
V
\end{array}\right)\right)=\left(\begin{array}{c}
U_{1} \\
V_{1}
\end{array}\right) \cdots\left(\begin{array}{c}
U_{m} \\
V_{m}
\end{array}\right)
$$


where all $\left(\begin{array}{c}U_{i} \\ V_{i}\end{array}\right)$ are minimal balanced pair and $\left(\begin{array}{c}\sigma_{1}(U) \\ \sigma_{2}(V)\end{array}\right)=\left(\begin{array}{c}U_{1} \ldots U_{m} \\ V_{1} \ldots V_{m}\end{array}\right)$. For more details, see [17] [18] [20].

\subsection{Intersection of Rauzy fractals.}

Definition 13. We say that a set is substitutive if it is the closure of the projection of the vertices of a stepped line associated with a substitution $\Sigma$ on a contracting space of the incidence matrix of $\Sigma$.

Proposition 1. [17] Let $\tau_{1}$ and $\tau_{2}$ be two unimodular Pisot irreducible substitutions with the same incidence matrix. We consider $\mathcal{R}_{\tau_{1}}$ and $\mathcal{R}_{\tau_{2}}$ their associated Rauzy fractals. We suppose that 0 is an exclusive inner point to $\mathcal{R}_{\tau_{1}}$. Then the set $\mathcal{E}$ has a nonempty interior and strictly positive Lebesgue measure.

If the substitutions $\tau_{1}$ and $\tau_{2}$ satisfy the Pisot conjecture, then the set $\mathcal{E}$ is also a Rauzy fractal associated to the substitution defined by the balanced pair algorithm. Sellami characterized the closure of the intersection of the interiors of two Rauzy fractals associated with two substitutions unimodular Pisot substitutions with the same incidence matrix, and more precisely as shown in the following Theorem:

Theorem 3.1. [18] Let $\tau_{1}$ and $\tau_{2}$ be two unimodular Pisot irreducible substitutions with the same incidence matrix. We consider $\mathcal{R}_{\tau_{1}}$ and $\mathcal{R}_{\tau_{2}}$ be their two associated Rauzy fractals. Suppose that 0 is an exclusive inner point of $\mathcal{R}_{\tau_{1}}$ and that one substitution of them satisfies the Pisot conjecture. We denote by $\mathcal{E}$ the closure of the intersection of the interiors of $\mathcal{R}_{\tau_{1}}$ and $\mathcal{R}_{\tau_{2}}$. Then $\mathcal{E}$ has a nonempty interior and is a substitutive set associated with the Pisot substitution $\Sigma$ obtained by the balanced pair algorithm, defined on the alphabet of minimal balanced pairs.

In next section, we study the symmetric intersection of Rauzy fractals associated with $k$-bonacci substitution.

\section{4. $k$-BONACCI INTERSECTION}

In this section we consider the substitution

$$
\sigma_{k}:\left\{\begin{array}{l}
1 \mapsto 12 \\
2 \mapsto 13 \\
\vdots \\
(k-1) \mapsto 1 k \\
k \mapsto 1
\end{array}\right.
$$

This substitution is irreducible of Pisot type [16]. We are interested in the study of the intersection of Rauzy fractals associated with $\sigma_{k}$ and $\sigma_{k}^{*}$, its reverse substitution. 
The two substitutions $\sigma_{k}$ and $\sigma_{k}^{*}$ have the same incidence matrix defined by

$$
M_{\sigma_{k}}=\left(\begin{array}{cccccccc}
1 & 1 & 1 & \cdots & 1 & 1 & 1 & 1 \\
1 & 0 & 0 & \cdots & 0 & 0 & 0 & 0 \\
0 & 1 & 0 & \cdots & 0 & 0 & 0 & 0 \\
& & & \ddots & & & & \\
0 & 0 & 0 & \cdots & 0 & 1 & 0 & 0 \\
0 & 0 & 0 & \cdots & 0 & 0 & 1 & 0
\end{array}\right) \quad \text { for } k \geq 2
$$

The characteristic polynomial of the incidence matrix $M_{\sigma_{k}}$ is

$$
P_{k}(x)=x^{k}-x^{k-1}-\cdots-x-1,
$$

for every $k \geq 2$ (see [23]).

In this paper we are interested in the intersection of Rauzy fractals associated with $\sigma_{k}$ and $\sigma_{k}^{*}$. We give a general formula for substitution associated with this intersection. Applying the balanced pair algorithm to these two substitutions, we characterize all minimal balanced pairs; we give exactly the cardinal of the set formed by all minimal balanced pairs; and we prove that the substitution describing the intersection has a regular form and that this substitution is over $\frac{k(k+1)}{2}$ letters.

First, we prove the following relation: for $k \geq 2$, we have

$$
\left(\sigma_{k}\right)^{k}(1)=\left(\sigma_{k}^{*}\right)^{k}(1) .
$$

In order to show this relation, we give the following Definition and Lemma:

Definition 14. We define a sequence of words $z_{k}$ on the infinite alphabet $\mathbb{N}$, for $k \geq 1$, with the following relation

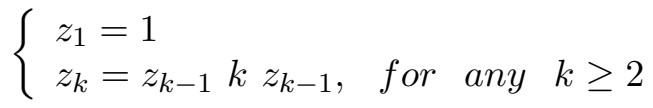

Remark 4.1. It is clear to see that the words $z_{k}$ are palindromes $\left(z_{k}^{*}=z_{k}\right)$.

Lemma 1. For any integer $k \geq 2$, we have:

$$
z_{k}=\sigma_{k}\left(z_{k-1}\right) 1 \text {. }
$$

Proof. By reasoning by induction on $k$, we have for $k=2, z_{2}=\sigma_{2}\left(z_{1}\right) 1$, since $z_{2}=121$ and $\sigma_{2}\left(z_{1}\right)=\sigma_{2}(1)=12$. Assuming that the equality holds up to the order $k$. Now, we show this property for the order $(k+1)$.

We have

$$
\begin{aligned}
z_{k+1} & =z_{k}(k+1) z_{k} \\
& =\sigma_{k}\left(z_{k-1}\right) 1(k+1) \sigma_{k}\left(z_{k-1}\right) 1
\end{aligned}
$$


Since $z_{k-1} \in\{1,2, \ldots, k-1\}^{*}$, we have $\sigma_{k+1}\left(z_{k-1}\right)=\sigma_{k}\left(z_{k-1}\right)$, because, by Definition of $\sigma_{k}$,

$$
\forall i \in\{1,2, \ldots, k-1\}^{*}, \text { we have } \sigma_{k+1}(i)=\sigma_{k}(i)=1(i+1) .
$$

Thus, we obtain

$$
\begin{aligned}
z_{k+1} & =\sigma_{k+1}\left(z_{k-1}\right) \sigma_{k+1}(k) \sigma_{k+1}\left(z_{k-1}\right) 1 \\
& =\sigma_{k+1}\left(z_{k-1} k z_{k-1}\right) 1 \\
& =\sigma_{k+1}\left(z_{k}\right) 1
\end{aligned}
$$

Lemma 2. Let $k \geq 2$ be a fixed integer.

Then, for any $j \in\{1, \ldots, k\}$, we have:

$$
P_{j}:\left\{\begin{array}{l}
\sigma_{k}^{j}(k+1-j)=z_{j} \\
\sigma_{k}^{j}(i-j)=z_{j} i,
\end{array} \quad \text { for } \quad j+1 \leq i \leq k\right.
$$

Proof. Reasoning by induction on $j$.

For $j=1$, we have

$$
P_{1}:\left\{\begin{array}{l}
\sigma_{k}(k)=1=z_{1} \\
\sigma_{k}(i-1)=1 i=z_{1} i, \quad \text { for } 2 \leq i \leq k
\end{array}\right.
$$

It is assumed that $P_{j}$ holds. For all $j \in\{1, \ldots k-1\}$ show $P_{j+1}$ holds.

$$
\begin{aligned}
\sigma_{k}^{j+1}(k+1-(j+1)) & =\sigma_{k}^{j+1}(k-j) \\
& =\sigma_{k}^{j}\left[\sigma_{k}(k-j)\right] \\
& =\sigma_{k}^{j}(1(k-j+1)) \\
& =\sigma_{k}^{j}(1) \sigma_{k}^{j}(k-j+1) \\
& =\sigma_{k}^{j}((j+1)-j) \sigma_{k}^{j}(k-j+1) \\
& =z_{j}(j+1) z_{j},
\end{aligned}
$$

which follows from $\sigma_{k}^{j}(1)=\sigma_{k}^{j}((j+1)-j)=z_{j}(j+1)$ and $\sigma_{k}^{j}(k-j+1)=$ $z_{j}$ by $P_{j}$.

Thus, we get

$$
\sigma_{k}^{j+1}(k+1-(j+1))=z_{j+1}
$$

For $j+2 \leq i \leq k$, we get

$$
\begin{aligned}
\sigma_{k}^{j+1}(i-(j+1)) & =\sigma_{k}^{j}\left[\sigma_{k}(i-j-1)\right] \\
& =\sigma_{k}^{j}(1(i-j)) \\
& =\sigma_{k}^{j}(1) \sigma_{k}^{j}(i-j) \\
& =\sigma_{k}^{j}((j+1)-j) \sigma_{k}^{j}(i-j) \\
& =z_{j}(j+1) z_{j} i \\
& =z_{j+1} i
\end{aligned}
$$


which follows from $\sigma_{k}^{j}(1)=\sigma_{k}^{j}((j+1)-j)=z_{j}(j+1)$ and $\sigma_{k}^{j}(i-j)=$ $z_{j} i$ by $P_{j}$.

Corollary 1. For $k \geq 2$, we have $\sigma_{k}^{k}(1)=z_{k}$.

The proof is easily obtained by applying Lemma(2) with $j=k$.

Lemma 3. For $k \geq 2$, we have

$$
\left(\sigma_{k}\right)^{k}(1)=\left(\sigma_{k}^{*}\right)^{k}(1)
$$

Proof. Since $z_{k}$ is a palindrome, then we have

$$
\left(\sigma_{k}^{*}\right)^{k}(1)=\left(\left(\sigma_{k}\right)^{k}(1)\right)^{*}=z_{k}^{*}=z_{k}=\left(\sigma_{k}\right)^{k}(1) .
$$

Definition 15. A minimal balanced pair $\left(\begin{array}{c}U \\ V\end{array}\right)$ is called a minimal balanced pair of type $j$ if the length of $U$ equals $j(i . e .,|U|=j=|V|$ ).

Then, we obtain the following Lemma:

Lemma 4. (1) Let $\left(\begin{array}{c}U \\ V\end{array}\right)$ be a minimal balanced pair obtained by applying the balanced pair algorithm to $\left(\sigma_{k}, \sigma_{k}^{*}\right)$. Then $\left(\begin{array}{c}U \\ V\end{array}\right)$ is of type $2^{j}$, with $0 \leq j \leq k-1$.

(2) The set $E_{j}$ of minimal balanced pairs of type $2^{j}$ obtained by applying the balanced pair algorithm to $\left(\sigma_{k}, \sigma_{k}^{*}\right)$ contain exactly $k-j$ elements.

Proof. (1) We apply the balanced pair algorithm with $\sigma_{k}$ and $\sigma_{k}^{*}$. The first minimal balanced pair that we can consider is the beginning of the two fixed points associated with $\sigma_{k}$ and $\left(\sigma_{k}^{*}\right)^{k}$ that will be $\left(\begin{array}{l}1 \\ 1\end{array}\right)$ of type $2^{0}$.

- For $i=1$ and $1+j \leq k$, we apply the balanced pair algorithm with $\sigma_{k}$ and $\sigma_{k}^{*}$ to the first minimal balanced pair. We represent the image of the first element of this pair by $\sigma_{k}$ and the second one by $\sigma_{k}^{*}$.

We obtain

$\left(\begin{array}{c}1 \\ 1\end{array}\right) \underset{\sigma_{k}, \sigma_{k}^{*}}{\longrightarrow}\left(\begin{array}{c}\sigma_{k}(1) \\ \sigma_{k}^{*}(1)\end{array}\right)=\left(\begin{array}{c}12 \\ 21\end{array}\right)=\left(\begin{array}{c}z_{1} 2 \\ 2 z_{1}\end{array}\right)$ by Definition(14).

We obtain a new balanced pair of type $2^{1}$. This new balanced pair $\left(\begin{array}{l}12 \\ 21\end{array}\right)$ is minimal because 2 appears only once.

The second step is to consider the same process with the new 
balanced pair $\left(\begin{array}{l}12 \\ 21\end{array}\right)$.

We consider the image of this balanced pair with the two substitutions $\sigma_{k}$ and $\sigma_{k}^{*}$ and we obtain

$\left(\begin{array}{c}12 \\ 21\end{array}\right) \underset{\sigma_{k}, \sigma_{k}^{*}}{\longrightarrow}\left(\begin{array}{c}\sigma_{k}(12) \\ \sigma_{k}^{*}(21)\end{array}\right)=\left(\begin{array}{c}\sigma_{k}^{2}(1) \\ \left(\sigma_{k}^{*}\right)^{2}(1)\end{array}\right)=\left(\begin{array}{c}1213 \\ 3121\end{array}\right)=\left(\begin{array}{c}z_{2} 3 \\ 3 z_{2}\end{array}\right)$ by Definition $(14)$.

We get a new different balanced pair of type $2^{2}$.

This new balanced pair $\left(\begin{array}{c}1213 \\ 3121\end{array}\right)$ is minimal because 3 appears only once.

We continue with the balanced pair algorithm up to the order $j$ with $\sigma_{k}$ and $\sigma_{k}^{*}$ with minimal balanced pair $\left(\begin{array}{l}1 \\ 1\end{array}\right)$.

$\left(\begin{array}{c}1 \\ 1\end{array}\right) \stackrel{\sigma_{k}^{j},\left(\sigma_{k}^{*}\right)^{j}}{\longrightarrow}\left(\begin{array}{c}\sigma_{k}^{j}(1) \\ \left(\sigma_{k}^{*}\right)^{j}(1)\end{array}\right)=\left(\begin{array}{c}z_{j}(1+j) \\ (1+j) z_{j}\end{array}\right)$ by Lemma $(2)$.

We get new balanced pairs of type $2^{j}$. We notice that $\left(\begin{array}{c}z_{j}(1+j) \\ (1+j) z_{j}\end{array}\right)$ is a minimal balanced pair because $(1+j)$ appears only once at the beginning and the end of the pair. We cannot decompose $\left(\begin{array}{c}z_{j}(1+j) \\ (1+j) z_{j}\end{array}\right)$ into minimal balanced pairs.

- For $1+j=k$, by applying the balanced pair algorithm to the order $j+1$, with $\sigma_{k}$ and $\sigma_{k}^{*}$ to the last minimal balanced pair $\left(\begin{array}{c}z_{j} k \\ k z_{j}\end{array}\right)$, we obtain a new different balanced pair $\left(\begin{array}{c}z_{j} k \\ k z_{j}\end{array}\right) \underset{\sigma_{k}, \sigma_{k}^{*}}{\longrightarrow}\left(\begin{array}{c}\sigma_{k}\left(z_{j} k\right) \\ \sigma_{k}^{*}\left(k z_{j}\right)\end{array}\right)=\left(\begin{array}{c}\sigma_{k}^{j+1}(1) \\ \left(\sigma_{k}^{*}\right)^{j+1}(1)\end{array}\right)=\left(\begin{array}{c}\sigma_{k}^{k}(1) \\ \left(\sigma_{k}^{*}\right)^{k}(1)\end{array}\right)=$ $\left(\begin{array}{c}z_{k} \\ z_{k}\end{array}\right)=\left(\begin{array}{l}z_{k-1} k z_{k-1} \\ z_{k-1} k z_{k-1}\end{array}\right)$ by Corollary $(1)$.

This last balanced pair can be decomposed with minimal balanced pairs $\left(\begin{array}{l}i \\ i\end{array}\right)$ of type $2^{0}$ because we obtain the same word.

We repeat this procedure for each of these new minimal balanced pairs $\left(\begin{array}{c}i \\ i\end{array}\right)$ of type $2^{0}$.

- For $1 \leq i \leq k$ and $i+j \leq k$, we apply the balanced pair algorithm with $\sigma_{k}$ and $\sigma_{k}^{*}$ to the minimal balanced pair $\left(\begin{array}{c}i \\ i\end{array}\right)$, of type $2^{0}$. We obtain

$\left(\begin{array}{c}i \\ i\end{array}\right) \underset{\sigma_{k}, \sigma_{k}^{*}}{\longrightarrow}\left(\begin{array}{c}\sigma_{k}(i) \\ \sigma_{k}^{*}(i)\end{array}\right)=\left(\begin{array}{c}1(i+1) \\ (i+1) 1\end{array}\right)=\left(\begin{array}{c}z_{1}(i+1) \\ (i+1) z_{1}\end{array}\right)$ by Definition(14). 
We get a new minimal balanced pair of type $2^{1}$. This new balanced pair $\left(\begin{array}{c}1(i+1) \\ (i+1) 1\end{array}\right)$ is minimal because $(i+1)$ appears only once.

The second step is to consider the same process with the new balanced pair $\left(\begin{array}{c}1(i+1) \\ (i+1) 1\end{array}\right)$. We take the image of this balanced pair with the two substitutions $\sigma_{k}$ and $\sigma_{k}^{*}$ and we obtain

$\left(\begin{array}{c}1(i+1) \\ (i+1) 1\end{array}\right) \stackrel{\sigma_{k}, \sigma_{k}^{*}}{\longrightarrow}\left(\begin{array}{c}\sigma_{k}(1(i+1)) \\ \sigma_{k}^{*}((i+1) 1)\end{array}\right)=\left(\begin{array}{c}\sigma_{k}^{2}(i) \\ \left(\sigma_{k}^{*}\right)^{2}(i)\end{array}\right)=\left(\begin{array}{c}121(i+2) \\ (i+2) 121\end{array}\right)=$
$\left(\begin{array}{c}z_{2}(i+2) \\ (i+2) z_{2}\end{array}\right)$ by Definition(14).

We get a new different balanced pair of type $2^{2}$.

This new balanced pair $\left(\begin{array}{c}121(i+2) \\ (i+2) 121\end{array}\right)$ is minimal because $(i+$

2) appears only once.

We continue with the balanced pair algorithm up to the order $j$ with $\sigma_{k}$ and $\sigma_{k}^{*}$ to the minimal balanced pair $\left(\begin{array}{c}i \\ i\end{array}\right)$.

$\left(\begin{array}{c}i \\ i\end{array}\right) \stackrel{\sigma_{k}^{j},\left(\sigma_{k}^{*}\right)^{j}}{\longrightarrow}\left(\begin{array}{c}\sigma_{k}^{j}(i) \\ \left(\sigma_{k}^{*}\right)^{j}(i)\end{array}\right)=\left(\begin{array}{c}z_{j}(i+j) \\ (i+j) z_{j}\end{array}\right)$ by Lemma(2).

We get a new balanced pairs of type $2^{j}$. We notice that $\left(\begin{array}{c}z_{j}(i+j) \\ (i+j) z_{j}\end{array}\right)$

is a minimal balanced pair because $(i+j)$ appears only once at the beginning and the end of the pair. We cannot decompose $\left(\begin{array}{c}z_{j}(i+j) \\ (i+j) z_{j}\end{array}\right)$ into minimal balanced pair.

- For $i+j=k$, by applying the balanced pair algorithm to the order $j+1$, with $\sigma_{k}$ and $\sigma_{k}^{*}$ with minimal balanced pair $\left(\begin{array}{c}z_{j} k \\ k z_{j}\end{array}\right)$, we obtain a new different balanced pair

$$
\begin{aligned}
& \left(\begin{array}{c}
z_{j} k \\
k z_{j}
\end{array}\right) \underset{\sigma_{k}, \sigma_{k}^{*}}{\longrightarrow}\left(\begin{array}{c}
\sigma_{k}\left(z_{j} k\right) \\
\sigma_{k}^{*}\left(k z_{j}\right)
\end{array}\right)=\left(\begin{array}{c}
\sigma_{k}^{j+1}(i) \\
\left(\sigma_{k}^{*}\right)^{j+1}(i)
\end{array}\right)=\left(\begin{array}{c}
\sigma_{k}^{k-i+1}(i) \\
\left(\sigma_{k}^{*}\right)^{k-i+1}(i)
\end{array}\right)= \\
& \left(\begin{array}{c}
z_{k-i+1} \\
z_{k-i+1}
\end{array}\right)=\left(\begin{array}{cc}
z_{k-i}(k-i+1) & z_{k-i} \\
z_{k-i}(k-i+1) & z_{k-i}
\end{array}\right) \text { by } \operatorname{Lemma}(2) \text {. }
\end{aligned}
$$

This last balanced pair can be decomposed with minimal balanced pairs of type $2^{0}$ because we obtain the same word.

Finally, the balanced pair algorithm terminates. We obtain all the minimal balanced pairs.

These pairs are: $\left(\begin{array}{c}z_{j}(i+j) \\ (i+j) z_{j}\end{array}\right)$ of type $2^{j}$.

(2) For $1 \leq i+j \leq k, 0 \leq j \leq k-1$ and $1 \leq i \leq k-j$ and for a given $j, i$ takes $k-j$ values. Therefore, there are $(k-j)$ pairs of type $2^{j}$, 
i.e.,

$$
\sharp E_{j}=k-j .
$$

This Lemma will permit us to obtain the cardinal exactly of the set formed by all minimal balanced pairs and to obtain a general formula for the intersection substitution associated with $\sigma_{k}$ and $\sigma_{k}^{*}$.

Using Theorem 3.1 in the case of the substitutions $\sigma_{k}$ and $\sigma_{k}^{*}$, we obtain the following main result:

Theorem 4.1. Let $\sigma_{k}$ and $\sigma_{k}^{*}$ be two substitutions defined as follows:

$$
\sigma_{k}:\left\{\begin{array}{l}
1 \mapsto 12 \\
2 \mapsto 13 \\
\vdots \\
(k-1) \mapsto 1 k \\
k \mapsto 1
\end{array} \quad \text { and } \quad \sigma_{k}^{*}:\left\{\begin{array}{l}
1 \mapsto 21 \\
2 \mapsto 31 \\
\vdots \\
(k-1) \mapsto k 1 \\
k \mapsto 1
\end{array}\right.\right.
$$

Let $\mathcal{R}_{\sigma_{k}}$ and $\mathcal{R}_{\sigma_{k}^{*}}$ be the respective Rauzy fractals. Then, the closure of the intersection of the interior of $\mathcal{R}_{\sigma_{k}}$ and $\mathcal{R}_{\sigma_{k}^{*}}$ has a nonempty interior.

Furthermore, it is a substitutive set associated to the substitution $\Sigma_{k}$ obtained by the balanced pair algorithm. The substitution $\Sigma_{k}$ is defined over the alphabet $E=\left\{A_{i j}: 1 \leq i, \quad 0 \leq j, \quad i+j \leq k\right\}$ of cardinal $\frac{k(k+1)}{2}$. The substitution $\Sigma_{k}$ is defined by:

$\Sigma_{k}\left(A_{i j}\right)=A_{i(j+1)}$ if $i+j<k$

$\Sigma_{k}\left(A_{i j}\right)=Z_{j+1}$ if $i+j=k$, where $Z_{1}=A_{10}$ and $Z_{j+1}=Z_{j} A_{(j+1) 0} Z_{j}$.

Proof. We describe the balanced pair algorithm and we obtain explicitly the substitution $\Sigma_{k}$ defined on $\frac{k(k+1)}{2}$ letters for $k \geq 2$.

We define $A_{i j}=\left(\begin{array}{c}z_{j}(i+j) \\ (i+j) z_{j}\end{array}\right)$ the minimal balanced pair of type $2^{j}$, obtained by Lemma 4 for $i \in\{1, \cdots, k\}$ and $j \in\{0, \cdots, k-1\}$.

We apply the balanced pair algorithm with $\sigma_{k}$ and $\sigma_{k}^{*}$. The first minimal balanced pair that we can consider is the beginning of the two fixed points associated with $\sigma_{k}$ and $\left(\sigma_{k}^{*}\right)^{k}$ that will be $\left(\begin{array}{l}1 \\ 1\end{array}\right)$.

- For $i=1$ and $1+j \leq k$, we apply the balanced pair algorithm with $\sigma_{k}$ and $\sigma_{k}^{*}$ to the first minimal balanced pair. We represent the image of the first element of this pair by $\sigma_{k}$ and the second one by $\sigma_{k}^{*}$. We obtain:

$$
\left(\begin{array}{c}
1 \\
1
\end{array}\right) \underset{\sigma_{k}, \sigma_{k}^{*}}{\longrightarrow}\left(\begin{array}{c}
\sigma_{k}(1) \\
\sigma_{k}^{*}(1)
\end{array}\right)=\left(\begin{array}{c}
12 \\
21
\end{array}\right)=\left(\begin{array}{c}
z_{1} 2 \\
2 z_{1}
\end{array}\right) \text { by Definition(14). }
$$


We denote by $A_{10}$ the minimal balanced pair $\left(\begin{array}{l}1 \\ 1\end{array}\right) \cdot A_{10}$ is a balanced pair of type $2^{0}$. Then, by $A_{11}$ the minimal balanced pair $\left(\begin{array}{l}12 \\ 21\end{array}\right), A_{11}$ is only a balanced pair of type $2^{1}$.

This new balanced pair $A_{11}$ is minimal because 2 appears only once. We deduce the relation $A_{10} \mapsto A_{11}$.

The second step is to consider the same process with the new balanced pair $A_{11}=\left(\begin{array}{l}12 \\ 21\end{array}\right)$.

We consider the image of this balanced pair with the two substitutions $\sigma_{k}$ and $\sigma_{k}^{*}$ and we obtain

$\left(\begin{array}{c}12 \\ 21\end{array}\right) \underset{\sigma_{k}, \sigma_{k}^{*}}{\longrightarrow}\left(\begin{array}{c}\sigma_{k}(12) \\ \sigma_{k}^{*}(21)\end{array}\right)=\left(\begin{array}{c}\sigma_{k}^{2}(1) \\ \left(\sigma_{k}^{*}\right)^{2}(1)\end{array}\right)=\left(\begin{array}{c}1213 \\ 3121\end{array}\right)=\left(\begin{array}{c}z_{2} 3 \\ 3 z_{2}\end{array}\right)$ by Definition $(14)$.

We obtain a new different balanced pair of type $2^{2}$ denoted $A_{12}$.

This new balanced pair $A_{12}$ is minimal because 3 appears only once. Hence we obtain $A_{11} \mapsto A_{12}$.

We continue with the balanced pair algorithm up to the order $j$, we take the image of $A_{1(j-1)}$ of type $2^{j-1}$.

$$
\left(\begin{array}{c}
1 \\
1
\end{array}\right) \underset{k}{\sigma_{k}^{j},\left(\sigma_{k}^{*}\right)^{j}}\left(\begin{array}{c}
\sigma_{k}^{j}(1) \\
\left(\sigma_{k}^{*}\right)^{j}(1)
\end{array}\right)=\left(\begin{array}{c}
z_{j}(1+j) \\
(1+j) z_{j}
\end{array}\right)=A_{1 j} \text { by Lemma(2). }
$$

We get a new different balanced pair of type $2^{j}$ denoted $A_{1 j}$. We notice that $A_{1 j}$ is a minimal balanced pair because $(1+j)$ appears only once at the beginning and the end of the pair. We cannot decompose $A_{1 j}$ into minimal balanced pair.

We deduce the relation $A_{1(j-1)} \mapsto A_{1 j}$.

- For $1+j=k$, by applying the balanced pair algorithm to the order $j+1$, with $\sigma_{k}$ and $\sigma_{k}^{*}$ to the minimal balanced pair $A_{k-j j}=\left(\begin{array}{c}z_{j} k \\ k z_{j}\end{array}\right)$, we obtain a new different balanced pair

$$
\begin{aligned}
& \left(\begin{array}{c}
z_{j} k \\
k z_{j}
\end{array}\right) \stackrel{\sigma_{k}, \sigma_{k}^{*}}{\longrightarrow}\left(\begin{array}{c}
\sigma_{k}\left(z_{j} k\right) \\
\sigma_{k}^{*}\left(k z_{j}\right)
\end{array}\right)=\left(\begin{array}{c}
\sigma_{k}^{j+1}(1) \\
\left(\sigma_{k}^{*}\right)^{j+1}(1)
\end{array}\right)=\left(\begin{array}{c}
\sigma_{k}^{k}(1) \\
\left(\sigma_{k}^{*}\right)^{k}(1)
\end{array}\right)= \\
& \left(\begin{array}{c}
z_{k} \\
z_{k}
\end{array}\right)=\left(\begin{array}{c}
z_{k-1} k z_{k-1} \\
z_{k-1} k z_{k-1}
\end{array}\right) \text { by Corollary }(1) .
\end{aligned}
$$

This last balanced pair can be decomposed with minimal balanced pairs of type $2^{0}$ because we obtain the same word.

We deduce the relation

$$
A_{1 j} \mapsto Z_{j+1} \text {, where } Z_{1}=A_{10} \text { and } Z_{j+1}=Z_{j} A_{(j+1) 0} Z_{j} \text {. }
$$

We repeat this procedure for each of these new minimal balanced pairs $\left(\begin{array}{c}i \\ i\end{array}\right)$ of type $2^{0}$. 
- For $1 \leq i \leq k$ and $i+j \leq k$, we apply the balanced pair algorithm with $\sigma_{k}$ and $\sigma_{k}^{*}$ to the minimal balanced pair of type $2^{0}, A_{i 0}=\left(\begin{array}{c}i \\ i\end{array}\right)$. We obtain:

$\left(\begin{array}{c}i \\ i\end{array}\right) \underset{\sigma_{k}, \sigma_{k}^{*}}{\longrightarrow}\left(\begin{array}{c}\sigma_{k}(i) \\ \sigma_{k}^{*}(i)\end{array}\right)=\left(\begin{array}{c}1(i+1) \\ (i+1) 1\end{array}\right)=\left(\begin{array}{c}z_{1}(i+1) \\ (i+1) z_{1}\end{array}\right)$ by Definition(14).

We get a new minimal balanced pair of type $2^{1}$. We denote it by $A_{i 1}=\left(\begin{array}{c}1(i+1) \\ (i+1) 1\end{array}\right)$.

This new balanced pair $A_{i 1}$ is minimal because $(i+1)$ appears only once.

We deduce the relation $A_{i 0} \mapsto A_{i 1}$.

The second step is to consider the same process with the new balanced pair $A_{i 1}$.

We consider the image of this balanced pair with the two substitutions $\sigma_{k}$ and $\sigma_{k}^{*}$ and we obtain

$\left(\begin{array}{c}1(i+1) \\ (i+1) 1\end{array}\right) \stackrel{\sigma_{k}, \sigma_{k}^{*}}{\longrightarrow}\left(\begin{array}{c}\sigma_{k}(1(i+1)) \\ \sigma_{k}^{*}((i+1) 1)\end{array}\right)=\left(\begin{array}{c}\sigma_{k}^{2}(i) \\ \left(\sigma_{k}^{*}\right)^{2}(i)\end{array}\right)=\left(\begin{array}{c}121(i+2) \\ (i+2) 121\end{array}\right)=$ $\left(\begin{array}{c}z_{2}(i+2) \\ (i+2) z_{2}\end{array}\right)$ by Definition(14).

We get a new different balanced pair of type $2^{2}$ denoted $A_{i 2}$.

This new balanced pair $A_{i 2}$ is minimal because $(i+2)$ appears only once.

Hence we obtain $A_{i 1} \mapsto A_{i 2}$.

We continue with the balanced pair algorithm up to the order $j$, we take the image of $A_{i(j-1)}$ of type $2^{j-1}$

$\left(\begin{array}{c}i \\ i\end{array}\right) \stackrel{\sigma_{k}^{j},\left(\sigma_{k}^{*}\right)^{j}}{\longrightarrow}\left(\begin{array}{c}\sigma_{k}^{j}(i) \\ \left(\sigma_{k}^{*}\right)^{j}(i)\end{array}\right)=\left(\begin{array}{c}z_{j}(i+j) \\ (i+j) z_{j}\end{array}\right)=A_{i j}$ by Lemma $(2)$.

We obtain a new different balanced pair $A_{i j}$ of type $2^{j}$.

We notice that $A_{i j}$ is a minimal balanced pair because $(i+j)$ appears only once at the beginning and the end of the pair. We cannot decompose $A_{i j}$ into minimal balanced pair.

We deduce the relation $A_{i(j-1)} \mapsto A_{i j}$.

- For $i+j=k$, by applying the balanced pair algorithm to the order $j+1$, with $\sigma_{k}$ and $\sigma_{k}^{*}$ to the minimal balanced pair $A_{k-j j}=\left(\begin{array}{c}z_{j} k \\ k z_{j}\end{array}\right)$, we obtain a new different balanced pair

$$
\begin{aligned}
& \left(\begin{array}{c}
z_{j} k \\
k z_{j}
\end{array}\right) \stackrel{\sigma_{k}, \sigma_{k}^{*}}{\longrightarrow}\left(\begin{array}{c}
\sigma_{k}\left(z_{j} k\right) \\
\sigma_{k}^{*}\left(k z_{j}\right)
\end{array}\right)=\left(\begin{array}{c}
\sigma_{k}^{j+1}(i) \\
\left(\sigma_{k}^{*}\right)^{j+1}(i)
\end{array}\right)=\left(\begin{array}{c}
\sigma_{k}^{k-i+1}(i) \\
\left(\sigma_{k}^{*}\right)^{k-i+1}(i)
\end{array}\right)= \\
& \left(\begin{array}{l}
z_{k-i+1} \\
z_{k-i+1}
\end{array}\right)=\left(\begin{array}{c}
z_{k-i}(k-i+1) \\
z_{k-i}(k-i+1) \\
z_{k-i}
\end{array}\right) \text { by } \operatorname{Lemma}(2) \text {. }
\end{aligned}
$$

This last balanced pair can be decomposed with minimal balanced 
pairs of type $2^{0}$ because we obtain the same word.

We deduce the relation

$$
A_{i j} \mapsto Z_{j+1} \text {, where } Z_{1}=A_{10} \text { and } Z_{j+1}=Z_{j} A_{(j+1) 0} Z_{j} \text {. }
$$

Finally, the balanced pair algorithm terminates. We obtain all the minimal balanced pairs.

We define the set of all minimal balanced pairs as follows:

$$
E=\left\{A_{i j}: 1 \leq i, \quad 0 \leq j, \quad i+j \leq k\right\} .
$$

From Lemma 4 , we have $\sharp E_{j}=k-j$ which permits us to calculate the cardinal of the set $E$ formed by all minimal balanced pairs. This cardinal will be shown as follows:

$$
\sharp E=\sum_{j=0}^{k-1} \sharp E_{j}=\sum_{j=0}^{k-1} k-j=\frac{k(k+1)}{2} .
$$

Thus, we obtain the substitution $\Sigma_{k}$ defined over the set formed by all minimal balanced pairs $E$ by a general formula as follows:

$$
\Sigma_{k}= \begin{cases}A_{i j}=A_{i(j+1)} & \text { if } i+j<k, \\ A_{i j}=Z_{j+1} & \text { if } i+j=k, \text { where } Z_{1}=A_{10} \text { and } Z_{j+1}=Z_{j} A_{(j+1) 0} Z_{j} .\end{cases}
$$

\section{Examples}

In this section, we compute $\Sigma_{k}$ explicitly for small values of $k$.

\section{Example 1}

For $k=2$, we consider two substitutions $\sigma_{2}$ and $\sigma_{2}^{*}$ defined as follows:

$$
\sigma_{2}:\left\{\begin{array}{l}
1 \mapsto 12 \\
2 \mapsto 1
\end{array} \quad \text { and } \quad \sigma_{2}^{*}:\left\{\begin{array}{l}
1 \mapsto 21 \\
2 \mapsto 1
\end{array}\right.\right.
$$

$\sigma_{2}$ is the Fibonacci substitution and $\sigma_{2}^{*}$ its reversed substitution.

The two substitutions $\sigma_{2}$ and $\sigma_{2}^{*}$ have the same incidence matrix defined by $M_{\sigma_{2}}=\left(\begin{array}{cc}1 & 1 \\ 1 & 0\end{array}\right)$.

The fixed points of $\sigma_{2}$ and $\left(\sigma_{2}^{*}\right)^{2}$ beginning with $u=1211212112112 \ldots$ and $v=1212112121121 \ldots$

We describe the balanced pair algorithm with $\sigma_{2}$ and $\sigma_{2}^{*}$ to the first minimal balanced pair $\left(\begin{array}{l}1 \\ 1\end{array}\right)$. We represent the image of the first element of this pair by $\sigma_{2}$ and the second one by $\sigma_{2}^{*}$. Then, we obtain $\left(\begin{array}{l}1 \\ 1\end{array}\right) \stackrel{\sigma_{2}, \sigma_{2}^{*}}{\longrightarrow}\left(\begin{array}{l}12 \\ 21\end{array}\right)$ and 
we denote by $A_{10}$ the minimal balanced pair $\left(\begin{array}{l}1 \\ 1\end{array}\right)$ and by $A_{11}$ the minimal balanced pair $\left(\begin{array}{l}12 \\ 21\end{array}\right)$. Hence, it follows that

$$
A_{10} \mapsto A_{11}
$$

The second step is to apply the two substitutions $\sigma_{2}$ and $\sigma_{2}^{*}$ to $A_{11}=$ $\left(\begin{array}{l}12 \\ 21\end{array}\right)$.

We obtain $\left(\begin{array}{c}12 \\ 21\end{array}\right) \underset{\sigma_{2}, \sigma_{2}^{*}}{\longrightarrow}\left(\begin{array}{c}121 \\ 121\end{array}\right)$.

The obtained balanced pair can be decomposed with minimal balanced pairs as:

$$
\left(\begin{array}{l}
1 \\
1
\end{array}\right)\left(\begin{array}{l}
2 \\
2
\end{array}\right)\left(\begin{array}{l}
1 \\
1
\end{array}\right)
$$

Then, the new minimal balanced pair $\left(\begin{array}{l}2 \\ 2\end{array}\right)$ appears and we denote it by $A_{20}=\left(\begin{array}{l}2 \\ 2\end{array}\right)$.

Therefore, the image of the letter $A_{11}$ is $A_{10} A_{20} A_{10}$.

We continue with this algorithm where we apply the two substitutions $\sigma_{2}$ and $\sigma_{2}^{*}$ to $A_{20}=\left(\begin{array}{l}2 \\ 2\end{array}\right)$ and we obtain $\left(\begin{array}{l}1 \\ 1\end{array}\right)$, which has already been considered.

Hence, we obtain $A_{20} \mapsto A_{10}$.

Now, in total, we obtain an alphabet $E$ on three symbols:

$$
E=\left\{A_{10}=\left(\begin{array}{l}
1 \\
1
\end{array}\right), A_{11}=\left(\begin{array}{l}
12 \\
21
\end{array}\right), A_{20}=\left(\begin{array}{l}
2 \\
2
\end{array}\right)\right\} .
$$

For fixed $j$, we define the set $E_{j}$ of minimal balanced pair of the type $2^{j}$ as follows:

For $k=2$, we have $j \in\{0,1\}$ and $i \in\{1,2\}$.

For $j=0$, we have

$$
E_{0}=\left\{A_{10}=\left(\begin{array}{l}
1 \\
1
\end{array}\right), A_{20}=\left(\begin{array}{l}
2 \\
2
\end{array}\right)\right\} .
$$

And for $j=1$, we have

$$
E_{1}=\left\{A_{11}=\left(\begin{array}{c}
12 \\
21
\end{array}\right)\right\}
$$

So, for a given $j, i$ takes $(2-j)$ values. Therefore, there are $(2-j)$ pairs of type $2^{j}$, i.e.,

$$
\sharp E_{j}=2-j .
$$


Thus,

$$
\sharp E=\sum_{j=0}^{1} \sharp E_{j}=\sum_{j=0}^{1} 2-j=\frac{2(2+1)}{2}=3 .
$$

We can define the substitution $\Sigma_{2}$ as:

$$
\Sigma_{2}=\left\{\begin{aligned}
A_{10} & \mapsto A_{11} \\
A_{11} & \mapsto A_{10} A_{20} A_{10} \\
A_{20} & \mapsto A_{10}
\end{aligned}\right.
$$

The substitution $\Sigma_{2}$ generates all the common points of the two stepped lines associated with $\sigma_{2}$ and $\sigma_{2}^{*}$.

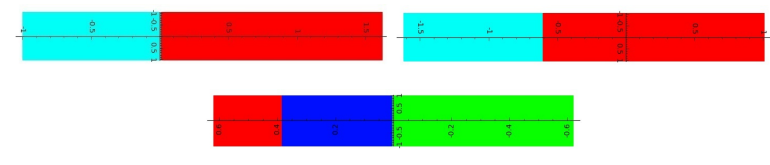

Figure 2. Rauzy fractals associated with $\sigma_{2}, \sigma_{2}^{*}$ and $\Sigma_{2}$.

The incidence matrix of $\Sigma_{2}$ is $M_{\Sigma_{2}}=\left(\begin{array}{lll}0 & 2 & 1 \\ 1 & 0 & 0 \\ 0 & 1 & 0\end{array}\right)$.

The determinant of $M_{\Sigma_{2}}$ is 1 . So the substitution $\Sigma_{2}$ is unimodular. The characteristic polynomial of $\sigma_{2}$ is $x^{2}-x-1$. The characteristic polynomial is associated with the substitution $\Sigma_{2}$ is $(x+1)\left(x^{2}-x-1\right)$.

This substitution is a primitive reducible Pisot .

\section{Example 2}

For $k=3$, we consider the Tribonacci substitution $\sigma_{3}$ and its reversed substitution $\sigma_{3}^{*}$ defined as:

$$
\sigma_{3}:\left\{\begin{aligned}
& 1 \mapsto 12 \\
& 2 \mapsto 13 \\
& 3 \mapsto 1
\end{aligned} \quad \text { and } \quad \sigma_{3}^{*}:\left\{\begin{array}{l}
1 \mapsto 21 \\
2 \mapsto 31 \\
3 \mapsto 1
\end{array}\right.\right.
$$

The two substitutions $\sigma_{3}$ and $\sigma_{3}^{*}$ have the same incidence matrix defined by $M_{\sigma_{3}}=\left(\begin{array}{lll}1 & 1 & 1 \\ 1 & 0 & 0 \\ 0 & 1 & 0\end{array}\right)$.

The fixed points of $\sigma_{3}$ and $\left(\sigma_{3}^{*}\right)^{3}$ beginning with $u=1213121121312 \ldots$ and $v=1213121213121 \cdots$.

When we apply the balanced pair algorithm to the two substitutions $\sigma_{3}$ and $\sigma_{3}^{*}$, we get the following balanced pairs $E$ : 


$$
\begin{aligned}
& A_{10}=\left(\begin{array}{l}
1 \\
1
\end{array}\right), A_{11}=\left(\begin{array}{l}
12 \\
21
\end{array}\right), A_{12}=\left(\begin{array}{l}
1213 \\
3121
\end{array}\right), A_{20}=\left(\begin{array}{l}
2 \\
2
\end{array}\right), A_{30}= \\
& \left(\begin{array}{l}
3 \\
3
\end{array}\right), A_{21}=\left(\begin{array}{l}
13 \\
31
\end{array}\right) .
\end{aligned}
$$

For fixed $j$, we define the set $E_{j}$ of minimal balanced pair of the type $2^{j}$ as follows:

For $k=3$, we have $j \in\{0,1,2\}$ and $i \in\{1,2,3\}$.

For $j=0$, we have

$$
E_{0}=\left\{A_{10}=\left(\begin{array}{l}
1 \\
1
\end{array}\right), A_{20}=\left(\begin{array}{l}
2 \\
2
\end{array}\right), A_{30}=\left(\begin{array}{l}
3 \\
3
\end{array}\right)\right\} .
$$

For $j=1$, we have

$$
E_{1}=\left\{A_{11}=\left(\begin{array}{l}
12 \\
21
\end{array}\right), A_{21}=\left(\begin{array}{l}
13 \\
31
\end{array}\right)\right\} .
$$

And for $j=2$, we have

$$
E_{2}=\left\{A_{12}=\left(\begin{array}{l}
1213 \\
3121
\end{array}\right)\right\} .
$$

So for a given $j, i$ takes $(3-j)$ values. Therefore, there are $(3-j)$ pairs of type $2^{j}$, i.e.,

$$
\sharp E_{j}=3-j .
$$

Thus,

$$
\sharp E=\sum_{j=0}^{2} \sharp E_{j}=\sum_{j=0}^{2} 3-j=\frac{3(3+1)}{2}=6 .
$$

Thus, we obtain the substitution $\Sigma_{3}$ for intersection on 6 symbols defined as:

$$
\Sigma_{3}:\left\{\begin{array}{l}
A_{10} \mapsto A_{11} \\
A_{11} \mapsto A_{12} \\
A_{12} \mapsto A_{10} A_{20} A_{10} A_{30} A_{10} A_{20} A_{10} \\
A_{20} \mapsto A_{21} \\
A_{21} \mapsto A_{10} A_{20} A_{10} \\
A_{30} \mapsto A_{10}
\end{array}\right.
$$

The substitution $\Sigma_{3}$ generates all the common points of the two stepped lines associated with $\sigma_{3}$ and $\sigma_{3}^{*}$.

$$
\text { The incidence matrix of } \Sigma_{3} \text { is } M_{\Sigma_{3}}=\left(\begin{array}{cccccc}
0 & 0 & 4 & 0 & 2 & 1 \\
1 & 0 & 0 & 0 & 0 & 0 \\
0 & 1 & 0 & 0 & 0 & 0 \\
0 & 0 & 2 & 0 & 1 & 0 \\
0 & 0 & 0 & 1 & 0 & 0 \\
0 & 0 & 1 & 0 & 0 & 0
\end{array}\right) \text {. }
$$

The determinant of $M_{\Sigma_{3}}$ is 1 . So the substitution $\Sigma_{3}$ is unimodular. 


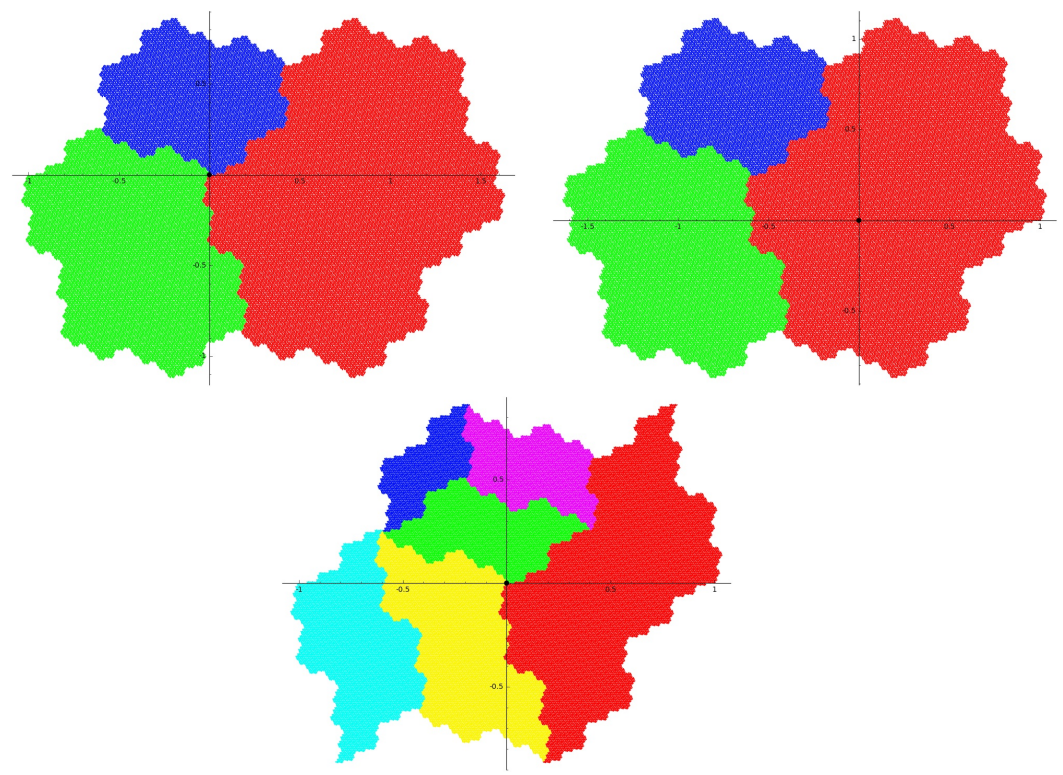

Figure 3. Rauzy fractals associated with $\sigma_{3}, \sigma_{3}^{*}$ and $\Sigma_{3}$.

The characteristic polynomial of $\sigma_{3}$ is $x^{3}-x^{2}-x-1$. The characteristic polynomial is associated with the substitution $\Sigma_{3}$ is $\left(x^{3}-x^{2}-x-1\right)\left(x^{3}+x^{2}+x-1\right)$. This substitution is a primitive reducible Pisot . 


\section{REFERENCES}

[1] Akiyama S., Loridant B. Boundary parametrization of self-affine tiles. Journal of Mathematics Society of Japan 2011; 63: 525-579.

[2] Arnoux P. Un exemple de semi-conjugaison entre un échange d'intervalles est une rotation sur le tore, Bull. Soc. Math. France 1988; 116: 489-500.

[3] Arnoux P., Ito S. Pisot substitutions and Rauzy fractals. Bull. Belg. Math. Soc. Simon Stevin 2001; 8: 181-207.

[4] Barge M., Diamond B. Coincidence for substitutions of Pisot type. Bull. Soc. Math. France 2002; 130: 619-626.

[5] Barge M., Kwapisz J. Geometric theory of unimodular Pisot substitutions. Am J Math 2006; 128: 1219-1282.

[6] Berthé V., Rigo M, editors. Combinatorics, Automata and Number Theory. Encyclopedia of Mathematics and its Applications, Book 135. Cambridge, UK: Cambridge University Press, 2010.

[7] Canterini V., Siegel A. Geometric representation of substitutions of Pisot type. T Am Math Soc 2001; 353: 5121-5144.

[8] Ei H., Ito S., Rao H. Atomic surfaces, tiling and coincidences II, reducible case. Ann Inst Fourier Grenoble 2006; 56: 2285-2313.

[9] FOGG P., Substitutions in dynamics, arithmetics and combinatorics, Lecture Notes in Mathematics, 1794, Springer-Verlag, Berlin, 2002.

[10] Holton C., Zamboni L. Geometric realization of substitutions. Bull Soc Math France 1998; 126: 149-179.

[11] Ito S., Kimura M. On the Rauzy fractal, Japan J. Ind. Appl. Math 1991; 8: 461-486.

[12] Lee JY., Moody RV., Solomyak B. Diffraction and multi-dimensional substitution systems. Annales Henri Poincaré 2002; 3: 1002-1018.

[13] Livshits AN. On the spectra of adic transformations of Markov compacta. Russian Math Surveys 1987; 42: 222-223.

[14] Pytheas Fogg N. Substitutions in Dynamics, Arithmetics and Combinatorics. Lecture Notes in Mathematics, Vol. 1974. Berlin, Germany: Springer, 2002.

[15] Queffélec M. Substitution dynamical systems-spectral analysis. Lecture Notes in Mathematics, 1294. Springer-Verlag, Berlin, 1987.

[16] Rauzy G. Nombres algébriques et substitutions. Bull Soc Math France 1982; 110: 147-178.

[17] Sellami T. Geometry of the common dynamics of Pisot substitutions with the same incidence matrix. C R Math Acd Sci Paris 2010; 348: 1005-1008.

[18] Sellami T. Common dynamics of two Pisot substitutions with the same incidence matrix. Publ Math Debrecen 2012; 81: 41-63.

[19] Sellami T. Balanced pair algorithm for a class of cubic substitutions. Turkish Journal of Mathematics 2015; 39: 91-102.

[20] Sellami T., Sirvent VF. Symmetric Intersections of Rauzy Fractals. Quaestiones Mathematicae, Vol. 39, Iss. 3, 2016; 289-305.

[21] Siegel A., Thuswaldner JM. Topological Properties of Rauzy Fractal. Marseille, France: Mémoires de la SMF, 2009.

[22] Sing B., Sirvent VF. Geometry of the common dynamics of flipped Pisot substitutions. Monatshefte fur Mathematik 2008; 155: 431-448.

[23] Sirvent VF. Properties of Geometrical Realizations of Substitutions associated to a Family of Pisot Numbers. Ph.D. Thesis. University of Warwick, 1993.

[24] Sirvent VF. The Common dynamics of the Tribonacci substitution, Bull. Belg. Math. Soc. Simon Stevin 2000; 7: 571-582.

[25] Sirvent VF., Wang Y. Self-affine tilings via substitution dynamical systems and Rauzy fractals. Pacific. J. Math 2002; 206: 465-485. 
[26] Sirvent VF. The Arnoux semi-conjugacy is Hölder continuous. Journal of Mathematical Analysis and Applications 2001; 259: 357-367.

[27] Sirvent VF. Relationships between the Dynamical Systems Associated to the Rauzy Substitutions. Theoretical Computer Science 1996; 164, 41-57.

[28] Sirvent VF., Solomyak B. Pure discrete spectrum for one dimensional substitution systems of Pisot type. Cand. Math. Bulletin 2002; 45: 697-710.

[29] Sirvent VF., Solomyak B. Pure discrete spectrum for one dimensional substitution systems of Pisot type. Canadien Math Bulletin 2002; 45: 697-710.

[30] Solomyak B. On the spectral theory of adic transformations. Advances in Soviet Mathematics 1992; 9: 217-230.

Sfax University, Faculty of sciences of Sfax, Department of mathematics, Route Soukra BP 802, 3018 Sfax, Tunisia.

E-mail address: hamdi.ammar.lfm@hotmail.fr

Aix-Marseille University, Institut de mathématiques de Marseille, 163 AvENUE DE LUMiny 13009 MARseille, FRANCE.

E-mail address: julien.cassaigne@math.cnrs.fr

Sfax University, Faculty of sciences of Sfax, Department of mathematics, Route Soukra BP 802, 3018 Sfax, Tunisia.

E-mail address: tarek.sellami.math@gmail.com 